\title{
The solar radius in the EUV during the cycle XXIII
}

\author{
C. G. Giménez de Castro $^{1}$, A. C. Varela Saraiva ${ }^{1,2}$, J. E. R. Costa ${ }^{2}$, and C. L. Selhorst ${ }^{1,2}$ \\ 1 Centro de Rádio Astronomia e Astrofísica Mackenzie, R. da Consolação 896, 01302-907 São Paulo, SP, Brazil \\ e-mail: guigue@craam.mackenzie.br \\ 2 Instituto Nacional de Pesquisas Espaciais, São José dos Campos, Brazil
}

Received 19 June 2007 / Accepted 27 September 2007

\section{ABSTRACT}

\begin{abstract}
Aims. We aim to determine the solar transition region and coronal radius at EUV wavelengths and its time evolution during Solar Cycle XXIII.

Methods. We use daily 30.4 and $17.1 \mathrm{~nm}$ images obtained by the Extreme Ultraviolet Imager (EIT) aboard the SoHO satellite and derive the solar radius by fitting a circle to the limb brightness ring.

Results. The weighted mean of the temporal series gives $\left(9677^{\prime \prime} 56 \pm 00^{\prime} 04\right)$ and $\left(9699^{\prime} 54 \pm 0{ }^{\prime} 02\right)$ at 30.4 and $17.1 \mathrm{~nm}$ respectively. No significant correlation was found with the solar cycle at either of the two wavelengths.

Conclusions. Since the temperature formation of the $30.4 \mathrm{~nm}$ line is between (60-80) $10^{3} \mathrm{~K}$ (Transition Region), the obtained result is greater than that derived from present optical atmospheric models. On the contrary, this height is compatible with radio models.
\end{abstract}

Key words. Sun: chromosphere - Sun: transition region - Sun: corona - Sun: UV radiation

\section{Introduction}

The solar atmospheric levels at which most of the different waveband brightness are produced have been analyzed by many authors to understand how the energy flow may affect the local equilibrium. Although there is a global hydrostatic equilibrium produced by an almost stable photospheric level, the total solar irradiance (the solar constant) varies (e.g. Stix 2002). The constancy or variation of the photospheric solar diameter has been a much debated subject. Coronal heating, however, is an indication that some energy is being deposited above the photosphere, modifying the equilibrium. The chromosphere and transition region height variations have been verified by solar radius measurements at radio wavelengths with a clear correlation to the solar constant changes (Costa et al. 1999).

Emilio et al. (2000) and Kuhn et al. (2004) have shown that the photospheric radius variation, if it exist, must be smaller than 15 mas during a whole solar cycle, therefore it can be taken as constant. Analysis in other frequencies of the electromagnetic spectrum have been carried out, mainly in the radio domain. Selhorst et al. (2004) have reviewed previous results and determined the solar radius variation at $17 \mathrm{GHz}$ using maps from the Nobeyama Radioheliograph (NoRH) with a space resolution of $5^{\prime \prime}$. They found a good correlation between the mean radius and the sunspot number but an anti-correlation between the polar radius and the sunspot number.

At UV frequencies there are very few previous works, with short temporal series analysis. In one of these, Zhang et al. (1998) used EIT full Sun images and concluded that: $i$ ) the solar disk is prolate (the same conclusion was reached by Auchère et al. 1998) and ii) the chromospheric $30.4 \mathrm{~nm}$ limb is significantly higher than the limb at 17.1, 19.5 and $28.4 \mathrm{~nm}$ wavelengths, arguing that spicules can contribute to the increase of the chromospheric / Transition Region (TR) height

The determination of the solar atmosphere layer's height is relevant to the formulation of atmospheric models. Many of these models are based on optical spectral analysis. The density and temperature dependence on height are derived by fitting a predicted spectrum to the observed one, e.g. Vernazza et al. (1973, 1976, 1981); Fontenla et al. (1990, 1991, 1993, 2002). All of these models obtain a TR height between 1700 and $2300 \mathrm{~km}$ above the photosphere. On the contrary, some $\mathrm{H} \alpha$ observations show a TR at around $5000 \mathrm{~km}$ above the photosphere (e.g. Zirin 1996). At microwaves, the solar radius has even larger values $\sim 10^{4} \mathrm{~km}$ (e.g. Selhorst et al. 2004). Zirin (1996) argued that the hydrostatic assumption of the atmospheric models is responsible for the disagreement between predictions and observations. On the other hand, Selhorst et al. (2005) claim that spicules are sufficient to increase the height of the solar limb when a model with a brightness temperature $T_{\mathrm{b}} \sim 11000 \mathrm{~K}$ at $17 \mathrm{GHz}$ and plasma densities few times $10^{10} \mathrm{~cm}^{-3}$ is used.

In this work we report the determination of the chromospheric/TR and coronal solar radius and their variation during the solar cycle XXIII using full Sun images of the Extreme Ultraviolet Imager Telescope (EIT) aboard the Solar Heliospheric Observatory (SoHO) obtained with the passband filters centered at $30.4 \mathrm{~nm}$ and $17.1 \mathrm{~nm}$. Our determination is based on the limb bright ring seen in both wavelengths. Since the $30.4 \mathrm{~nm}$ He line forms at around few times $10^{4} \mathrm{~K}$ we compare our observations with the microwave determinations of Selhorst et al. (2004). We present the method and results in Sect. 2, while in Sect. 3 we analyze the obtained time series and discuss our results.

\section{The determination of the limb bright ring}

The EIT is one of the 12 instruments aboard the SoHO satellite (Moses et al. 1997). It obtains images in four wavelengths by means of passband filters centered on the coronal lines $\lambda=17.1 \mathrm{~nm}\left(\mathrm{Fe}\right.$ IX,X, $\left.T \sim 10^{6}, \mathrm{~K}\right), \lambda=19.5 \mathrm{~nm}$ (Fe XII, $\left.T \sim 1.4 \times 10^{6} \mathrm{~K}\right)$ and $\lambda=28.4 \mathrm{~nm}\left(\mathrm{Fe} \mathrm{XV}, T \sim 2.1 \times 10^{6} \mathrm{~K}\right)$. 
$17.1 \mathrm{~nm}$
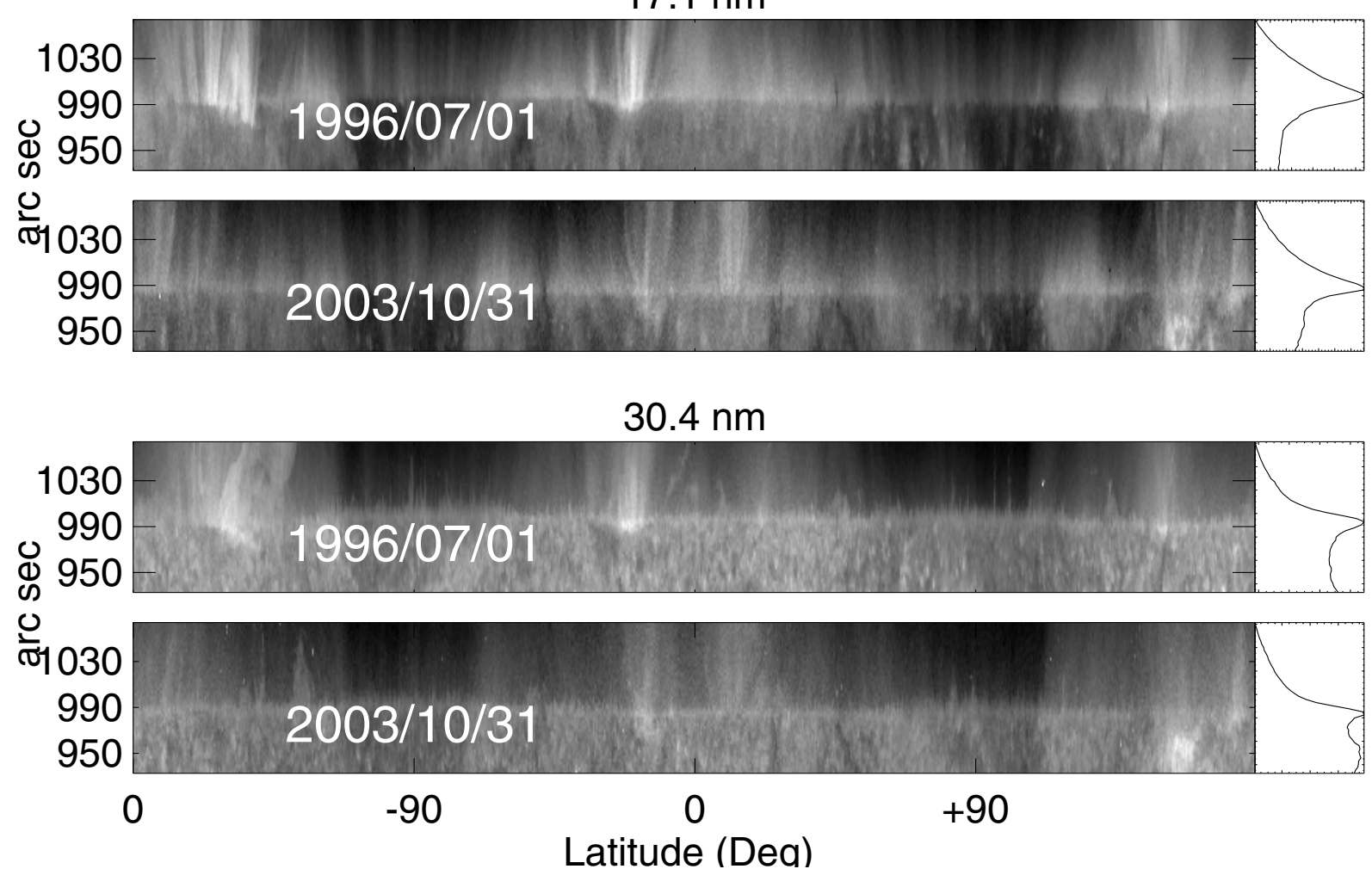

Fig. 1. EUV limb brightening. Images show the limb during different solar cycle phases. Right panels represent the mean limb profile.

The fourth wavelength range corresponds to a chromospheric / TR line, $\lambda=30.4 \mathrm{~nm}$ (He II Ly $\alpha, T \sim 6-8 \times 10^{4} \mathrm{~K}$ ).

We chose images centered at the wavelengths $\lambda=17.1 \mathrm{~nm}$ and $\lambda=30.4 \mathrm{~nm}$. The first line corresponds to the blending of $\mathrm{Fe}$ $\mathrm{X}, \lambda=17.106 \mathrm{~nm}$ and Fe IX, $\lambda=17.056 \mathrm{~nm}$ (Cowan $\&$ Peacock 1965). Both lines are optically thin, therefore they are most intense where the column depth is greater (e.g. Withbroe 1970). The maximum of the Fe IX, X emission is a pure coronal feature which can be used to determine a solar coronal radius that can be compared with radio continuum observations. Furthermore, since the emission is optically thin, changes in the coronal energy input may affect this radius and therefore this definition allows us to investigate the coronal heating during a solar cycle. On the other hand, the maximum of the radial profile is very sharp (see Fig. 2) making the radius determination very precise. Our criterion for this line is different to the one used by Zhang et al. (1998) who determines the occulting limb of the EUV iron lines in a way which is equivalent to the Auchère et al. (1998) limb definition as the inflection point of the radial profile. We note, though, that this occulting or blocking limb corresponds to a chromospheric layer which is investigated in this paper by means of the helium images.

The formation of the He II line is more controversial. There is an ambiguity between the predicted and observed intensities and there is a discussion about its mechanism (e.g. Jordan 1975; Andretta et al. 2003). In general the line is considered optically thick and formed in a thin layer (Pietarila \& Judge 2004) also resulting in a limb bright ring. Although this ring is not intense and clearly defined as the coronal one, it can be seen in the EIT images during the whole solar cycle. The line is contaminated by the coronal $\lambda=30.3 \mathrm{~nm}$ Si XI line, which may have a relative intensity of up to $20 \%$ above the disk brightening (Auchère 2000). Since Si XI is a coronal line, we expect that it will increase the radius determination, although there is no easy way to quantify the effect. We believe that since it is a weak line it will produce a small shift masked by the other uncertainties.

We define the solar radius as the semi-distance between the diametrically opposed maxima of the limb bright points of the rings. This definition allows us to determine the radius with an automatic methodology which is explained below.

\subsection{Limb extraction and radius determination}

Each image was corrected using standard procedures included in the Solar Software package for the EIT instrument. We use the Canny (1986) algorithm to obtain a first approach to the limb ring; a circular fitting to this ring gives us a disk center determination better than the one found in the image header. Once the center is well determined, radial profiles are extracted every half a degree in polar angle. The limb brightening of each profile is fitted to a special function $f(r)$ that depends on the wavelength band:

$17.1 \mathrm{~nm}: \quad f(r)=a_{\circ} \operatorname{atan}\left(\left(a_{1}-r\right) a_{2}\right)+a_{3}+a_{4} r^{a_{5}} \exp \left(-\left|r-r_{m}\right| a_{6}\right)$, $30.4 \mathrm{~nm}: \quad f(r)=a_{\circ} \exp \left(-\left(r-r_{m}\right)^{2} / 2 a_{1}^{2}\right)+a_{2}+a_{3} r+a_{4} r^{2}$.

Fe IX,X limb brightening is better represented by a cusp function while the arctangent is used to represent the transition between the scattered coronal light and the quiet sun. He II limb profiles do not have a clear peak and thus are not well represented by a cusp function, therefore we use a Gaussian to determine the mean brightest position of the limb. In this case a second degree polynomial represents the transition from the scattered light and the quiet sun. In both functions, $a_{i}$ and $r_{m}$ are the fitting parameters to be determined, the latter is the position of the limb maximum. The fit is carried out by means of the amoeba algorithm (Press et al. 2002), setting the optical radius 


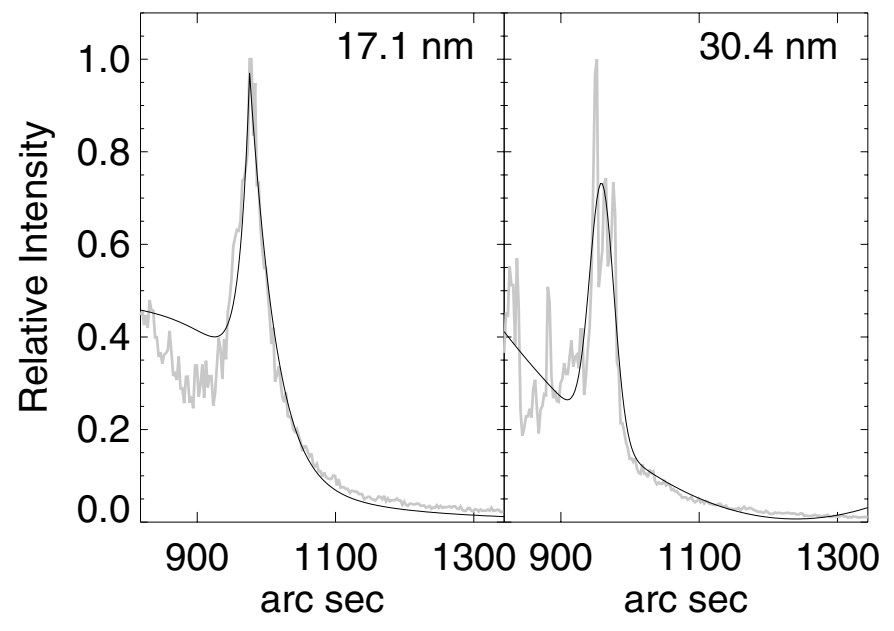

Fig. 2. Examples of limb fit. Left, a limb profile at $17.1 \mathrm{~nm}$ (gray) and the fit (black). Right, the same for the $30.4 \mathrm{~nm}$ limb profile.

as the lower limit of the fitting interval (see Fig. 2 for examples of the fits). We iteratively fit a circle to the set of $(x, y)$ ring positions, discarding at each step those that are outside the band $(R-1.8 \sigma, R+1.8 \sigma)$, with $R$ the circle radius and $\sigma$ the standard deviation of the fit. The iteration continues until it converges with all pairs inside the fitting band. To convert from the CCD pixel units to arc seconds ("), we use the plate scale determined during a 1999 Mercury transit by Auchère \& Artzner (2004), namely $2.627 \pm 0.001$ arc sec pixel ${ }^{-1}$. This figure was later confirmed with a new transit of Mercury in 2003 (Artzner, personal communication). The resulting size is corrected to $1 \mathrm{AU}$ using the satellite distance found in the image header.

\subsection{Results}

Figure 3 shows the time evolution of the solar 17.1 and $30.4 \mathrm{~nm}$ radii. Heights are relative to the photospheric radius at $1 \mathrm{AU}$, $R_{\odot}=959$ ' $^{\prime} 68=695489.2 \mathrm{~km}$. Points represent individual measurements, continuous curves are 28 day running means and the horizontal thick lines are the weighted means. There are some gaps in the data when the instrument stopped operation. Remarkable oscillations are observed before the gap at the end of 1998. An incorrect determination of the satellite distance, or a variation of the telescope focal distance produced by thermal oscillations can account for an incomplete data correction. Nonetheless it is not clear why the effect is so large at $30.4 \mathrm{~nm}$ and less significant at $17.1 \mathrm{~nm}$. Since we do not have certainty on the origin of the strong oscillations we do not use the data for the analysis before the long interruption of the instrument in 1998. In general, the mean uncertainty in radius determination is $1 . .7$, almost half a pixel, at $30.4 \mathrm{~nm}$ and $1^{\prime \prime}$, a third of a pixel, at $17.1 \mathrm{~nm}$. The larger uncertainty for the line of He II reveals its weaker intensity and its complex nature.

The very interesting harmonic variations of the solar limb during the cycle shown in Fig. 3 are still under analysis.

\section{Analysis and discussion}

Table 1 summarizes our results. The weighted means show a clear different height for the chromospheric He II and the coronal Fe IX,X lines. Even if we use the mean uncertainty of one measurement, the heights do not overlap. It is also clear that the chromosphere height is well above where present atmospheric models predict and near where Johannesson \& Zirin (1996)

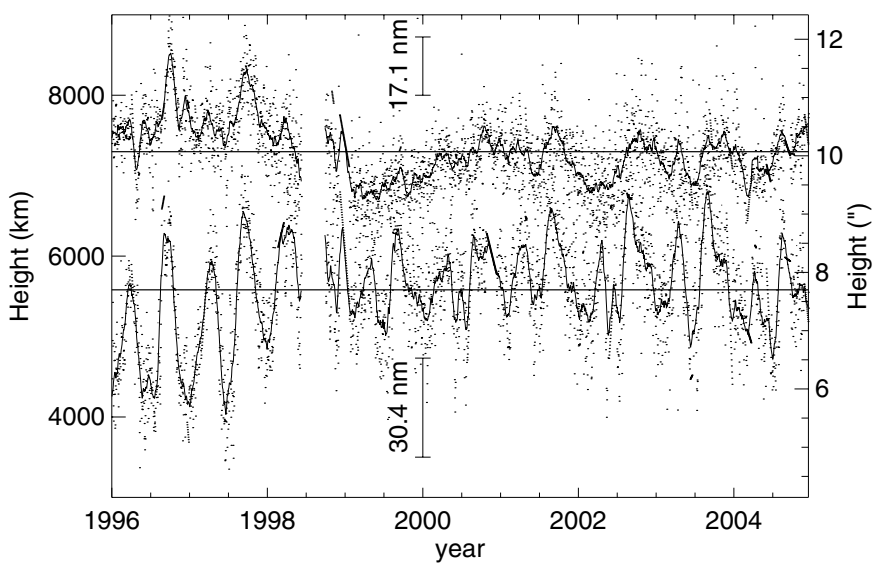

Fig. 3. Time evolution of the solar radii. Points represent individual measurements. Solid curves are 28 day running means at $17.1 \mathrm{~nm}$ (upper) and $30.4 \mathrm{~nm}$ (lower). Straight thick lines are the weighted mean values for the period 1999-2005. The vertical bars represent the mean uncertainty for one measurement.

Table 1. Solar radius and height over the photosphere.

\begin{tabular}{ccc}
\hline \hline & $\begin{array}{c}\text { Radius } \\
\left({ }^{\prime \prime}\right)\end{array}$ & $\begin{array}{c}\text { Height } \\
\mathrm{km}\end{array}$ \\
\hline He II & $967.56 \pm 0.04$ & $5710 \pm 30$ \\
Fe IX,X & $969.54 \pm 0.02$ & $7146 \pm 15$ \\
\hline
\end{tabular}

found it using $\mathrm{H} \alpha$ filtergrams and is comparable to Zhang et al. (1998).

Auchère et al. (1998) have shown that the Sun is 1 .'5 and 5" prolate at 17.1 and $30.4 \mathrm{~nm}$ respectively. Zhang et al. (1998) have also observed a similar prolatness of the EUV solar limb. Due to the noisy limb we did not fit an ellipse. We use instead a circle fit that is much simpler and faster. The ellipsoidal form is revealed after the average of over 400 images in Auchère et al. (1998). Therefore, our results represent an averaged value over the solar limb. Taking Auchere's results into account we can correct our determination of the equatorial radius by subtracting $2^{\prime \prime}$ and the polar radius by adding $3^{\prime \prime}$. In that way we obtain a $4130 \mathrm{~km}$ equatorial height and $7750 \mathrm{~km}$ polar height. Zhang et al. (1998) find $3100 \pm 1200 \mathrm{~km}$ and $6600 \pm 1200 \mathrm{~km}$ for the equatorial and polar heights respectively, in accordance with our results. On the other hand, we cannot compare our $17.1 \mathrm{~nm}$ height with that obtained in Zhang's work because of our different definition of coronal radius.

\subsection{Comparison with radio observations and solar indices variations}

The SSC atmospheric model proposed by Selhorst et al. (2005) predicts a solar radius of $966 . ' 5$ at $17 \mathrm{GHz}$. This microwave continuum emission comes from a solar atmospheric layer at a temperature of around $10^{4} \mathrm{~K}$, i.e. the chromosphere below the region where the Fe IX, X and He II are formed. Thus, the results of this work agree with that model. However, Selhorst et al. (2004) have determined a radius of around 975" from $17 \mathrm{GHz}$ NoRH maps. In Selhorst et al. (2005) the authors claim that the presence of spicules can explain the greater observed radius. Since typical spicule temperatures are between $0.5 \times 10^{4}$ and $2.5 \times 10^{4} \mathrm{~K}$ (see Sterling 2000, and references therein), their contribution to the EUV lines can be neglected and there is no contradiction 


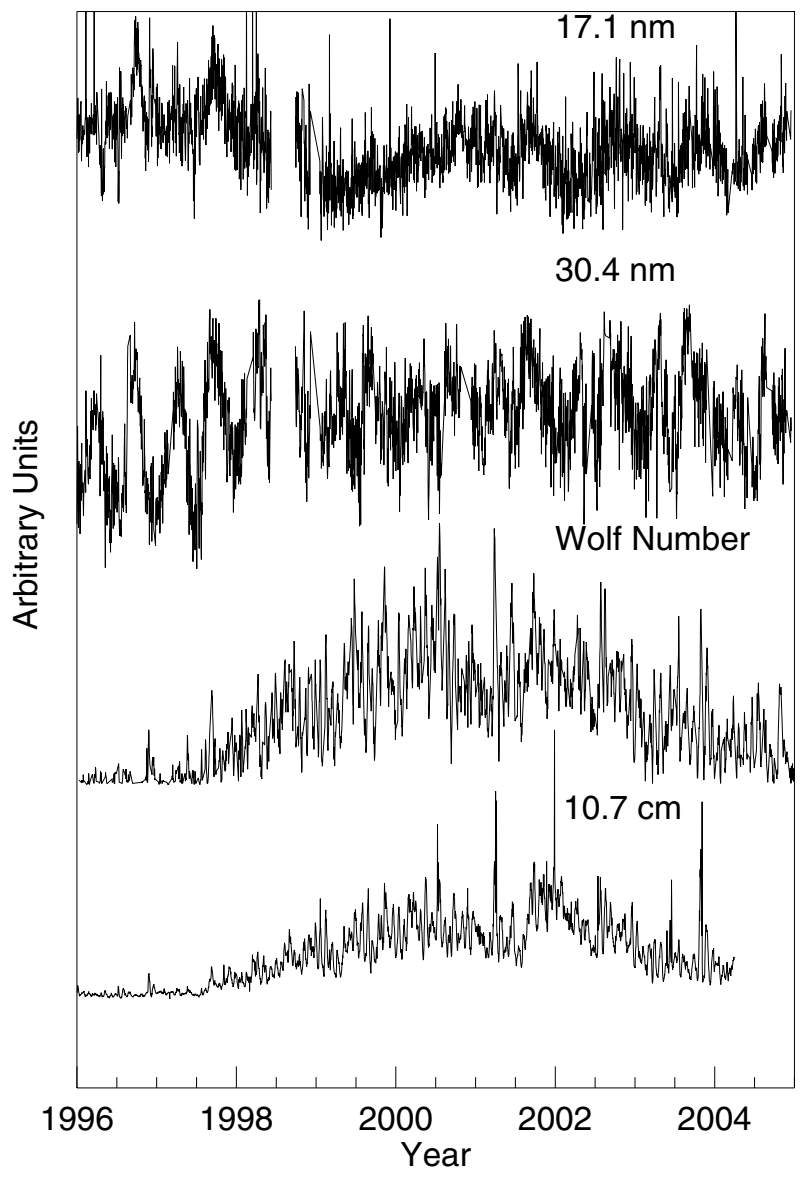

Fig. 4. EUV radius variations compared with solar cycle indices. From top to bottom: $17.1 \mathrm{~nm}$ radius, $30.4 \mathrm{~nm}$ radius, Wolf number and flux density at $10.7 \mathrm{~cm}$. All values are rescaled between $[-0.5,0.5]$ to facilitate the comparison.

Table 2. Cross correlation between our results of the solar radii and solar activity indicators. From left to right: solar radius at $17 \mathrm{GHz}$, total solar irradiance, Wolf number and flux density daily means at $10.7 \mathrm{~cm}$ (S-component).

\begin{tabular}{ccccc}
\hline \hline & $17 \mathrm{GHz}$ & Irr. & Wolf N. & $10.7 \mathrm{~cm}$ \\
\hline He II & +0.14 & +0.20 & +0.30 & -0.12 \\
Fe IX,X & -0.12 & -0.38 & -0.43 & -0.40 \\
\hline
\end{tabular}

in having the height of the $30.4 \mathrm{~nm}$ chromospheric line forming below the observed $17 \mathrm{GHz}$ continuum.

In Fig. 4 we plot our results together with the daily Wolf number and the daily mean flux density at $10.7 \mathrm{~cm}$ solar indices. For comparison we have rescaled the values in the band $[-0.5,0.5]$. The figure shows no clear indication of a close association between the radius and the solar indices. The cross correlation between the He II and Fe IX, X radius and the solar indices are shown in Table 2 . The near zero figures indicate that no correlation exists, although there may be some anticorrelation, of around -0.4 , between the coronal line and the solar indices. The real nature of such an anti-correlation remains to be confirmed. It is surprising that the solar activity does not change the solar EUV radius either at chromospheric or at coronal heights as it is observed, e.g. at $17 \mathrm{GHz}$ (Selhorst et al. 2005). The microwave continuum may come from a wide atmospheric layer receiving an important contribution from the spicules while a narrower layer is expected to form the EUV lines. Therefore it is possible that the variation in position of the EUV layers are not detectable with the present methodology and instrumentation.

Although we find no long term variations and the use of the running means erases changes with durations of the order of or less than 28 days, Fig. 3 shows fluctuations with a hundred day temporal scale that are more sensitive for the He II than for the Fe IX,X images. This variation deserves careful examination to determine wether it is an instrumental effect or a real solar phenomenon.

Acknowledgements. A.C.S. acknowledges support from the Fundação de Amparo à Pesquisa do estado de São Paulo (FAPESP) under grant No. 03/03500-2. The present project received partial support from Fundo Mackenzie de Pesquisa (Mackpesquisa). The authors are in debt to G. Artzner and M. Emilio for their fruitful discussions.

\section{References}

Andretta, V., Del Zanna, G., \& Jordan, S. D. 2003, A\&A, 400, 737 Auchère, F. 2000, Ph.D. Thesis, Universite Paris VI Auchère, F., \& Artzner, G. E. 2004, Sol. Phys., 219, 217

Auchère, F., Boulade, S., Koutchmy, S., et al. 1998, A\&A, 336, L57 Canny, J. 1986, IEEE Trans. Pattern Analysis and Machine Intelligence, 8, 679 Costa, J. E. R., Silva, A. V. R., Makhmutov, V. S., et al. 1999, ApJ, 520, L63 Cowan, R. D., \& Peacock, N. J. 1965, ApJ, 142, 390 Emilio, M., Kuhn, J. R., Bush, R. I., \& Scherrer, P. 2000, ApJ, 543, 1007 Fontenla, J. M., Avrett, E. H., \& Loeser, R. 1990, ApJ, 355, 700 Fontenla, J. M., Avrett, E. H., \& Loeser, R. 1991, ApJ, 377, 712 Fontenla, J. M., Avrett, E. H., \& Loeser, R. 1993, ApJ, 406, 319 Fontenla, J. M., Avrett, E. H., \& Loeser, R. 2002, ApJ, 572, 636 Johannesson, A., \& Zirin, H. 1996, ApJ, 471, 510 Jordan, C. 1975, MNRAS, 170, 429

Kuhn, J. R., Bush, R. I., Emilio, M., \& Scherrer, P. H. 2004, ApJ, 613, 1241 Moses, D., Clette, F., Delaboudinière, J.-P., et al. 1997, Sol. Phys., 175, 571 Pietarila, A., \& Judge, P. G. 2004, ApJ, 606, 1239

Press, W. H., T., V. W., A., T. S., \& P., F. S. 2002, Numerical recipes in C, the art of scientific computing, 2nd edn. (Cambridge University Press), 410 Selhorst, C. L., Silva, A. V. R., \& Costa, J. E. R. 2004, A\&A, 420, 1117 Selhorst, C. L., Silva, A. V. R., \& Costa, J. E. R. 2005, A\&A, 433, 365 Sterling, A. C. 2000, Sol. Phys., 196, 79

Stix, M. 2002, The Sun, 2nd edn. (Springer)

Vernazza, J. E., Avrett, E. H., \& Loeser, R. 1973, ApJ, 184, 605 Vernazza, J. E., Avrett, E. H., \& Loeser, R. 1976, ApJS, 30, 1

Vernazza, J. E., Avrett, E. H., \& Loeser, R. 1981, ApJS, 45, 635 Withbroe, G. L. 1970, Sol. Phys., 11, 42

Zhang, J., White, S. M., \& Kundu, M. R. 1998, ApJ, 504, L127

Zirin, H. 1996, Sol. Phys., 169, 313 